Honam Mathematical J. 35 (2013), No. 4, pp. 717-727

http://dx.doi.org/10.5831/HMJ.2013.35.4.717

\title{
AN EXTREMAL PROBLEM OF HOLOMORPHIC FUNCTIONS IN THE COMPLEX PLANE
}

\author{
Young-Bok Chung* And Byoung-IL PARK
}

\begin{abstract}
In this paper, we study on a higher order extremal problem relating the Ahlfors map associated to the pair of a finitely connected domain in the complex plane and a point there. We show the power of the Ahlfors map with some error term which is conformally equivalent maximizes any higher order derivative of holomorphic functions at the given point in the domain.
\end{abstract}

\section{Introduction}

It is well known (see [6]) that the power of the Riemann mapping function of a simply connected domain solves a higher order extremal problem as follows: if $\Omega$ is a simply connected domain in the plane such that each of the boundary components consists of more than one boundary point and $a \in \Omega$ is given, then for a fixed positive integer $m$, the power $g=\left(f_{a}\right)^{m}$ of the Riemann mapping function $f_{a}$ associated to the pair $(\Omega, a)$ solves uniquely the extremal problem

$$
\operatorname{Re} g^{(m)}(a)=\max \operatorname{Re} h^{(m)}(a)
$$

among holomorphic functions $h$ mapping $\Omega$ into the unit disc with a zero of order $m$ at $a$. On the other hand, Chung showed that there is no direct generalization of the above extremal problem for the Ahlfors map associated to the pair $(\Omega, a)$ in the case of finitely connected domain $\Omega$ (see [7]). However we can pose the same sort of question as finding such a solution and Chung also showed in [7] that a solution exists and is a proper holomorphic mapping of $\Omega$ onto the unit disc. Since any proper holomorphic mapping of finitely connected domain onto the unit

Received October 1, 2013. Accepted November 29, 2013.

2010 Mathematics Subject Classification. Primary 30C40.

Key words and phrases. extremal, Rieman mapping function, Ahlfors map, kernel function.

${ }^{*}$ Corresponding author 
disc can be written in terms of the classical kernel functions such as the Szegő kernel (see [5]), it is very important to solve the extremal problem in more general setting.

In this paper, we prove a theorem as a partial result with additional conditions for zeroes of the Ahlfors map which can arise in a natural way, and obtain another result on conformal invariance of the induced constants associated to the domain which seems to be very important in that we can change given domain into some easy domain so that we can find an extremal solution as desired.

Finally, we would like to thank the referees for proofreading this article and for making many corrections.

\section{Preliminaries and Some Notations}

Suppose that $\Omega$ is a finitely $n$-connected domain in the plane with $C^{\infty}$ smooth boundary. Let $T$ be denoted by the unit tangent vector function defined on the boundary $b \Omega$ with the standard orientation of positive direction. The space $C^{\infty}(b \Omega)$ of $C^{\infty}$ smooth functions on $b \Omega$ is equipped with the $L^{2}$ inner product

$$
<u, v>=\int_{b \Omega} u \bar{v} d s, \quad\left(u, v \in C^{\infty}(\mathrm{b} \Omega)\right),
$$

where $d s$ denotes the differential element of arc length. Let $L^{2}(b \Omega)$ be the Hilbert space completion of $C^{\infty}(b \Omega)$ with respect to the inner product $<u, v>$ and let $H^{2}(b \Omega)$ denote the classical Hardy space on associated to $\Omega$ which is the space of holomorphic functions on $\Omega$ with $L^{2}$-boundary values in $b \Omega$. Then there exists the orthogonal projection of $L^{2}(b \Omega)$ onto $H^{2}(b \Omega)$ called the Szegö projection denoted by $P: \mathrm{七}^{2}(b \Omega) \rightarrow H^{2}(b \Omega)$.

The reproducing kernel $S(z, \zeta)$ for the Szego" projection $P$ is called the Szegö kernel and $S(z, \zeta)$ is defined and $C^{\infty}$ smooth on $(\bar{\Omega} \times \bar{\Omega})-$ $\{(z, z) \mid z \in b \Omega\}$, it is holomorphic in $z \in \Omega$ for fixed $\zeta \in \bar{\Omega}$, and it is antiholomorphic in $\zeta \in \Omega$ for fixed $z \in \bar{\Omega}$.

The Garabedian kernel $L(z, \zeta)$ is defined as follows. Let $C_{\zeta}(z)$ denote the Cauchy kernel which is defined by the complex conjugate of $\frac{T(z)}{2 \pi i(z-\zeta)}$ for $z \in b \Omega, \zeta \in \Omega$. Note that if $f$ is a function in $H^{2}(b \Omega)$, then

$$
h(\zeta)=<h, C_{\zeta}>
$$


It is known (see [2], [4]) that the orthogonal decomposition of the kernel $C_{\zeta}$ has the form

$$
C_{\zeta}=P\left(C_{\zeta}\right)+\overline{P\left(\overline{C_{\zeta} T}\right) T}
$$

Observing $S(z, \zeta)=P\left(C_{\zeta}\right)(z)$, it follows that

$$
S(z, \zeta)=i \overline{L(z, \zeta)} \overline{T(z)}, \quad \zeta \in \Omega, z \in b \Omega,
$$

where

$$
L(z, \zeta)=\frac{1}{2 \pi(z-\zeta)}-i P\left(\overline{C_{\zeta} T}\right)(z)
$$

It is easy to see from (2.2) that for fixed $\zeta \in \Omega, L(z, \zeta)$ is a meromorphic function on $\Omega$ with a single simple pole at $z=\zeta$ having residue $\frac{1}{2 \pi}$ extending $C^{\infty}$ smoothly up to the boundary of $\Omega$. Notice that $L(z, \zeta)$ never vanishes for all $(z, \zeta) \in \bar{\Omega} \times \Omega$ with $z \neq \zeta$.

These two kernel functions above are closely related to the Ahlfors map in a multiply connected domain which is a generalization of the Riemann mapping function in a simply connected domain. Given a finitely connected $n$-connected domain $\Omega$ in the plane with $C^{\infty}$ smooth boundary and $a \in \Omega$, the Ahlfors map $f_{a}$ associated to the pair $(\Omega, a)$ is the unique solution to the extremal problem: among all holomorphic functions $h$ mapping $\Omega$ into the unit disc, find the one making $h^{\prime}(a)$ real-valued and as large as possible. It is well known that the function $f_{a}$ is an $n$-to one proper holomorphic covering map of $\Omega$ onto the unit disc and is related to the Szegö kernel and Garabedian kernel functions via the identity

$$
f_{a}(z)=\frac{S(z, a)}{L(z, a)}
$$

See [4].

Bell showed (see [4], [3]) that there exists a finite subset $A$ of $\Omega$ so that $S(z, a)$ has $n-1$ distinct simple zeroes in $\Omega$ for every point $a \in \Omega \backslash A$. $n-1$ zeroes in $\Omega$ of $S(z, a)$ are taken account into those of the Ahlfors map $f_{a}$ except at $z=a$. Note also that $S(z, a) \neq 0$ for $z \in b \Omega$ and $a \in \Omega$.

\section{Main results}

Now we can show that higher order derivatives of a holomorphic function of a given domain into the unit disc can be represented in terms of the Ahlfors map and the Garabedian kernel. 
Theorem 3.1. Let $n$ be a positive integer. Suppose that $\Omega$ is a finitely $n$-connected domain in the plane with $C^{\infty}$ smooth boundary. Let $a \in \Omega$ be fixed. Let $m \geq 2$ be a postive integer. Suppose that the Ahlfors map $f_{a}$ associated to the pair $(\Omega, a)$ has $n$ all distinct zeroes at $a, a_{1}, a_{2}, \cdots, a_{n-1}$ in $\Omega$.

If $h$ is a holomorphic function mapping $\Omega$ into the unit disc in the plane which extends $C^{\infty}$ smoothly to the boundary of $\Omega$, having $a$ as a zero of order $m$, then

$$
\begin{aligned}
& h^{(m)}(a)=-2 \pi i m ! f_{a}^{\prime}(a)^{m-1} \int_{b \Omega} \frac{L(z, a)^{2}}{f_{a}^{m-1}(z)} h(z) d z+ \\
& 4 \pi^{2} m(m-1) f_{a}^{\prime}(a)^{m-1} \sum_{j=1}^{n-1} \sum_{l=0}^{m-2}\left(\begin{array}{c}
m-2 \\
l
\end{array}\right)\left[f_{j}(z)^{1-m} L(z, a)^{2}\right]^{(l)}\left(a_{j}\right) h^{(m-2-l)}\left(a_{j}\right)
\end{aligned}
$$

where $f_{a}(z)=\left(z-a_{j}\right) f_{j}(z)$ near $z=a_{j}$.

Proof. For a fixed $a \in \Omega$, let $h$ be a holomorphic function mapping $\Omega$ into the unit disc which extends $C^{\infty}$ smoothly to the boundary of $\Omega$, having $a$ as a zero of order $m$. We define the function $\Phi$ by

$$
\Phi(z)=\frac{L(z, a)^{2}}{f_{a}^{m-1}(z)} h(z) .
$$

Then $\Phi$ is a meromorphic function in $\bar{\Omega}$ with poles(possibly) at $a, a_{1}, a_{2}$, $\cdots, a_{n-1}$.

Note that the Ahlfors map $f_{a}$ has a simple zero at $z=a$ and the Garabedian kernel $L(z, a)$ has a simple single pole at $z=a$ with residue $\frac{1}{2 \pi}$. It follows that the function $\Phi$ has a simple pole at $z=a$ with residue

$$
\operatorname{Res}(\Phi ; a)=\lim _{z \rightarrow a}(z-a) \frac{L(z, a)^{2}}{f_{a}^{m-1}(z)} h(z)=\frac{1}{4 \pi^{2} m ! f_{a}^{\prime}(a)^{m-1}} h^{(m)}(a) .
$$

On the other hand, for fixed $a \in \Omega$, since $L(z, a)$ never vanishes in $\Omega$ and $f_{a}$ has a simple zero at each $z=a_{j}$, the function $\Phi$ has a pole of order at most $m-1$ at $z=a_{j}$ with residue

$$
\operatorname{Res}\left(\Phi ; a_{j}\right)=\frac{1}{(m-2) !} \lim _{z \rightarrow a_{j}} \frac{d^{m-2}}{d z^{m-2}}\left[\frac{\left(z-a_{j}\right)^{m-1} L(z, a)^{2}}{f_{a}^{m-1}(z)} h(z)\right] .
$$

Letting $f_{a}(z)=\left(z-a_{j}\right) f_{j}(z)$ near $z=a_{j}$, we obtain the identity 


$$
\begin{aligned}
\operatorname{Res}\left(\Phi ; a_{j}\right) & =\frac{1}{(m-2) !} \lim _{z \rightarrow a_{j}} \frac{d^{m-2}}{d z^{m-2}}\left[f_{j}(z)^{1-m} L(z, a)^{2} h(z)\right] \\
& =\frac{1}{(m-2) !} \sum_{l=0}^{m-2}\left(\begin{array}{c}
m-2 \\
l
\end{array}\right)\left[f_{j}(z)^{1-m} L(z, a)^{2}\right]^{(l)}\left(a_{j}\right) h^{(m-2-l)}\left(a_{j}\right) .
\end{aligned}
$$

We now apply the residue theorem for the function $\Phi$ in $\Omega$ to get

$$
\begin{aligned}
& \frac{1}{4 \pi^{2} m ! f_{a}^{\prime}(a)^{m-1}} h^{(m)}(a)=\frac{1}{2 \pi i} \int_{b \Omega} \Phi(z) d z \\
& -\frac{1}{(m-2) !} \sum_{j=1}^{n-1} \sum_{l=0}^{m-2}\left(\begin{array}{c}
m-2 \\
l
\end{array}\right)\left[f_{j}(z)^{1-m} L(z, a)^{2}\right]^{(l)}\left(a_{j}\right) h^{(m-2-l)}\left(a_{j}\right),
\end{aligned}
$$

which proves the conclusion.

We can from Theorem 3.1 solve the higher order extremal problem when the class of holomorphic functions $h$ is restricted to the subclass with having $a_{j}$ as a zero.

Theorem 3.2. Let $n$ be a positive integer. Suppose that $\Omega$ is a finitely $n$-connected domain in the plane with $C^{\infty}$ smooth boundary. Let $a \in \Omega$ be fixed. Let $m \geq 2$ be a postive integer. Suppose that the Ahlfors map $f_{a}$ associated to the pair $(\Omega, a)$ has $n$ all distinct zeroes at $a, a_{1}, a_{2}, \cdots, a_{n-1}$ in $\Omega$.

Then the power $f_{a}^{m}$ of the Ahlfors map is the unique extremal function maximizing the quantity

$$
\operatorname{Re} h^{(m)}(a)
$$

among holomorphic functions $h$ mapping $\Omega$ into the unit disc which extends $C^{\infty}$ smoothly to the boundary of $\Omega$, having $a$ as a zero of order $m$ and having $a_{j}$ as a zero of order at least $m-1$ for each $j=1,2, \cdots, n-1$.

Proof. Let $h$ be a holomorphic function of $\Omega$ into the unit disc having the property in the statement as above. The second term of the identity in (3.1) vanishes and hence it follows from the property $|f(z)|=1$ on $b \Omega$ that

$$
\left|h^{(m)}(a)\right| \leq 2 \pi m ! f_{a}^{\prime}(a)^{m-1} \int_{b \Omega}\left|L(z, a)^{2}\right| d s .
$$


Note that $|L(z, a)|=|S(z, a)|$ on $b \Omega$ from the identity (2.1). It also follows from the reproducing property of the Szegö kernel that

$$
S(a, a)=\int_{b \Omega}\left|S(z, a)^{2}\right| d s .
$$

On the other hand, it is easy to see from (2.3) that

$$
f_{a}^{\prime}(a)=2 \pi S(a, a) .
$$

Finally we obtain from (3.5) that

$$
\left|h^{(m)}(a)\right| \leq m ! f_{a}^{\prime}(a)^{m}=\left.\frac{d^{m}}{d z^{m}} f_{a}^{m}\right|_{z=a}
$$

which shows that the power $f_{a}^{m}$ of the Ahlfors map is a solution of the extremal problem.

For the uniqueness of the solution, we prove by induction on the number $m$. The case of $m=2$ was proved in [6]. We assume that $m>2$ and suppose that the uniqueness has been proved for the case of $m-1$. Let $G$ be an extremal solution of (3.4) which is a holomorphic function mapping $\Omega$ into the unit disc which extends $C^{\infty}$ smoothly to $b \Omega$. Since $G$ has the point $a$ as a zero of order $m$ and the point $a_{j}$ as a zero of order at least $m-1$, the function $G / f_{a}$ has $a$ as a zero of order $m-1$ and $a_{j}$ as a zero of order at least $m-2$. Hence it follows that

$$
\left(\frac{G}{f_{a}}\right)^{(m-1)}(a)=(m-1) ! \frac{G^{(m)}(a)}{m !} / f_{a}^{\prime}(a)=\frac{G^{(m)}(a)}{m f_{a}^{\prime}(a)},
$$

which is, from the extremal property of $G$, greater than or equal to

$$
\frac{\left(f_{a}^{m}\right)^{(m)}(a)}{m f_{a}^{\prime}(a)}=\left(f_{a}^{m-1}\right)^{(m-1)}(a) .
$$

By the assumption of induction, it implies that

$$
\frac{G}{f_{a}}=f_{a}^{m-1},
$$

which proves the uniqueness of the extremal solution for the case of $m$ and hence we prove the theorem.

Now we show in fact that smoothness of the boundary of the given domain in Theorem 3.2 is unnecessary.

Theorem 3.3. Let $n$ be a natural number. Suppose that $\Omega$ is a finitely $n$-connected domain in the plane. Let $a \in \Omega$ be fixed. Let $m \geq 2$ be a postive integer. Suppose that the Ahlfors map $f_{a}$ associated to the pair $(\Omega, a)$ has $n$ all distinct zeroes at $a, a_{1}, a_{2}, \cdots, a_{n-1}$ in $\Omega$. 
Then the power $f_{a}^{m}$ of the Ahlfors map is the unique extremal function maximizing the quantity

$$
\operatorname{Re} h^{(m)}(a)
$$

among holomorphic functions $h$ mapping $\Omega$ into the unit disc having a as a zero of order $m$ and having $a_{j}$ as a zero of order at least $m-1$ for each $j=1,2, \cdots, n-1$.

Proof. It follows from the Riemann Mapping Therorem that there is a biholomorphic map $\varphi$ of $\Omega$ onto a bounded domain $\widetilde{\Omega}$ with $C^{\infty}$ smooth boundary(see [1], [8]). Since the collection of such functions $h$ is a normal family, an extremal function of the problem exists. Let $g$ be an extremal solution of (3.7). Note from the transformation formula of the Ahlfors map (3.13) that the Ahlfors map $\widetilde{f}_{\varphi(a)}$ associated to the pair $(\widetilde{\Omega}, \varphi(a))$ has $n$ all distinct zeroes at $\varphi(a), \varphi\left(a_{1}\right), \cdots, \varphi\left(a_{n-1}\right)$ in $\widetilde{\Omega}$. Suppose that $\widetilde{h}$ is a holomorphic function of $\widetilde{\Omega}$ into the unit disc which extends $C^{\infty}$ smoothly to the boundary of $\widetilde{\Omega}$, having $\varphi(a)$ as a zero of order $m$ and having $\varphi\left(a_{j}\right)$ as a zero of order at least $m-1, j=1,2, \cdots, n-1$. Since the function $h=\widetilde{h} \circ \varphi$ is a holomorphic function of $\Omega$ into the unit disc belonging to the family of (3.7), it follows from the extremal property of $g$ that

$$
\left|h^{(m)}(a)\right| \leq\left|g^{(m)}(a)\right| .
$$

It is thus easy to see from $h^{(m)}(a)=\widetilde{h}^{(m)}(\varphi(a)) \varphi^{\prime}(a)^{m}$ that the composition $\widetilde{g}=g \circ \varphi^{-1}$ solves the extremal problem

$$
\left|\widetilde{g}^{(m)}(\varphi(a))\right|=\max \left|\widetilde{h}^{(m)}(\varphi(a))\right|,
$$

which from the uniqueness of the solution of the smooth case(Theorem 3.2) implies that there is a constant $\lambda$ with $|\lambda|=1$ such that

$$
\widetilde{g}=\lambda\left(\tilde{f}_{\varphi(a)}\right)^{m} \text { on } \widetilde{\Omega}
$$

and thus

$$
g=\lambda\left(\widetilde{f}_{\varphi(a)}\right)^{m} \circ \varphi \text { on } \Omega .
$$

It follows from the transformation formula of the Ahlfors map (3.13) that the extremal function $g$ is a rotation of the power $f_{a}^{m}$ of the Ahlfors map and hence the positivity of $f_{a}^{\prime}(a)$ implies the identity $g=f_{a}^{m}$.

The following theorem generalizes Theorem 4.1 in [6] to any higher order extremal problem. In fact, we show the power of the Ahlfors map with some error term which is conformally equivalent maximizes any higher order derivative of holomorphic functions. 
Theorem 3.4. Let $n$ be a natural number. Suppose that $\Omega$ is a finitely $n$-connected domain in the plane with $C^{\infty}$ smooth boundary. Let $a \in \Omega$ be fixed. Let $m \geq 2$ be a postive integer. Suppose that the Ahlfors map $f_{a}$ associated to the pair $(\Omega, a)$ has $n$ all distinct zeroes at $a, a_{1}, a_{2}, \cdots, a_{n-1}$ in $\Omega$.

Then there exist non-negative real constants $c_{j l}, j=1,2, \cdots, n-$ $1, l=0,1, \cdots, m-2$ depending on $a$, which are conformally invariant under certain normalized conformal maps, such that if $h$ is a holomorphic function mapping $\Omega$ into the unit disc in the plane which extends $C^{\infty}$ smoothly to the boundary of $\Omega$, having $a$ as a zero of order $m$, then

$$
\left|h^{(m)}(a)\right| \leq\left(f_{a}^{m}\right)^{(m)}(a)\left(1+\sum_{j=1}^{n-1} \sum_{l=0}^{m-2} c_{j l}\left|h^{(m-2-l)}\left(a_{j}\right)\right|\right) .
$$

Proof. The key point of the proof is based on the identity (3.1) and the proof of Theorem 3.2. It follows from the proof of Theorem 3.2 that the absolute value of the first term of the right hand side in (3.1) is less than equal to

$$
\left(f_{a}^{m}\right)^{(m)}(a) .
$$

For $j=1,2, \cdots, n-1, l=0,1, \cdots, m-2$, we define

$$
c_{j l}=\left.\frac{4 \pi^{2}}{f_{a}^{\prime}(a)} \frac{1}{(m-l-2) ! l !} \frac{d^{l}}{d z^{l}}\left[\left(\frac{z-a_{j}}{f_{a}(z)}\right)^{m-1} L(z, a)^{2}\right]\right|_{z=a_{j}} .
$$

Then from (3.1), we obtain the identity (3.9) immediately.

We now prove the conformal invariance of the constants $c_{j l}$. Suppose that $\widetilde{\Omega}$ is a bounded domains with $C^{\infty}$ smooth boundary and $\varphi: \Omega \rightarrow \widetilde{\Omega}$ is a biholomorphic mapping between $\Omega$ and $\widetilde{\Omega}$. We denote $\widetilde{f}$ by the Ahlfors map associated to the pair $(\widetilde{\Omega}, \varphi(a))$. We also denote $\widetilde{S}(w, b)$ and $\widetilde{L}(w, b)$ by the Szegö kernel and the Garabedian kernel associated to the domain $\widetilde{\Omega}$, respectively. It is well known that the Szegö kernel and the Garabedian kernel functions transform under a biholomorphic map via the following formulas:

$$
\begin{aligned}
& S(z, a)=\sqrt{\varphi^{\prime}(z)} \overline{\sqrt{\varphi^{\prime}(a)}} \widetilde{S}(\varphi(z), \varphi(a)), \\
& L(z, a)=\sqrt{\varphi^{\prime}(z)} \overline{\sqrt{\varphi^{\prime}(a)}} \widetilde{L}(\varphi(z), \varphi(a)),
\end{aligned}
$$

where $\sqrt{\varphi^{\prime}}$ is one of the square roots of $\varphi^{\prime}$. 
In virtue of the identity

$$
f_{a}(z)=\frac{S(z, a)}{L(z, a)}
$$

it is easy to get the transformation formula for the Ahlfors map via

$$
f_{a}(z)=\frac{\overline{\sqrt{\varphi^{\prime}(a)}}}{\sqrt{\varphi^{\prime}(a)}} \tilde{f}(\varphi(z), \varphi(a)) .
$$

It follows from (3.13) that if the Ahlfors map $f_{a}$ has $n$ all distinct zeroes at $a, a_{1}, a_{2}, \cdots, a_{n-1}$ in $\Omega$, then the corresponding Ahlfors map $\widetilde{f}$ associated to $(\widetilde{\Omega}, \varphi(a))$ must also have $n$ all distinct zeroes at $\varphi(a), \varphi\left(a_{1}\right), \varphi\left(a_{2}\right)$, $\cdots, \varphi\left(a_{n-1}\right)$.

Now the constant associated to $(\widetilde{\Omega}, \varphi(a))$ corresponding to $c_{j l}$ is equal to

(3.14) $\widetilde{c}_{j l}=\left.\frac{4 \pi^{2}}{\widetilde{f}^{\prime}(\varphi(a))} \frac{1}{(m-l-2) ! l !} \frac{d^{l}}{d w^{l}}\left[\left(\widetilde{f}_{j}(w)\right)^{1-m} \widetilde{L}(w, \varphi(a))^{2}\right]\right|_{w=\varphi\left(a_{j}\right)}$,

where $\widetilde{f}(w)=\left(w-\varphi\left(a_{j}\right)\right) \widetilde{f}_{j}(w)$ near $w=\varphi\left(a_{j}\right)$. Thus, in order to compare the constant $c_{j l}$ with $\widetilde{c}_{j l}$, we need to compute derivatives of $f_{a}(z), \widetilde{f}(w), L(z, a), \widetilde{L}(w, \varphi(a))$. Differentiating both sides of (3.12) and inserting $\frac{L(z, a)}{\sqrt{\varphi^{\prime}(z)} \sqrt{\varphi^{\prime}(a)}}$ into $\widetilde{L}(\varphi(z), \varphi(a))$, we obtain

$$
\begin{aligned}
& \left.\frac{d}{d w} \widetilde{L}(w, \varphi(a))\right|_{w=\varphi(z)} \\
& =\frac{1}{\sqrt{\varphi^{\prime}(a)}}\left(-\frac{1}{2} \frac{\varphi^{\prime \prime}(z)}{\sqrt{\varphi^{\prime}(z)^{5}}} L(z, a)+\frac{1}{\sqrt{\varphi^{\prime}(z)^{3}}} \frac{d}{d z} L(z, a)\right) .
\end{aligned}
$$

More generally, for $l=0,1, \cdots, m-2$, there exist constants $d_{l j}, j=$ $0,1, \cdots, l$, positive integers $\alpha_{l j n}$ and constants $m_{l j n}$ with

$$
1 \leq \alpha_{l j n} \leq l+1, j=0,1, \cdots, l, n=1,2, \cdots, l+1
$$

such that

$$
\begin{aligned}
\left.\frac{d^{l}}{d w^{l}} \widetilde{L}(w, \varphi(a))\right|_{w=\varphi(z)}= & \frac{1}{\sqrt{\varphi^{\prime}(a)}} \sum_{j=0}^{l} d_{l j}\left(\frac{d^{\alpha_{l j 1}}}{d z^{\alpha_{l j 1}}} \varphi(z)\right)^{m_{l j 1}} \\
& \cdots\left(\frac{d^{\alpha_{l j(l+1)}}}{d z^{\alpha_{l j(l+1)}}} \varphi(z)\right)^{m_{l j(l+1)}} \frac{d^{j}}{d z^{j}} L(z, a) .
\end{aligned}
$$


In particular, notice that $d_{l l}=1$ and

$$
\left(\frac{d^{\alpha_{l l 1}}}{d z^{\alpha_{l l 1}}} \varphi(z)\right)^{m_{l l 1}} \cdots\left(\frac{d^{\alpha_{l l(1+1)}}}{d z^{\alpha_{l l(1+1)}}} \varphi(z)\right)^{m_{l l(l+1)}}=\varphi^{\prime}(z)^{-\frac{2 l+1}{2}} .
$$

It also follows from (3.13) that there exist constants $e_{l j}, j=0,1, \cdots, l$, positive integers $\beta_{l j n}$ and constants $n_{l j p}$ with

$$
1 \leq \beta_{l j p} \leq l, j=1, \cdots, l, p=1,2, \cdots, l
$$

satisfying

$$
e_{l l}=1,\left(\frac{d^{\beta_{l l 1}}}{d z^{\beta_{l l 1}}} \varphi(z)\right)^{n_{l l 1}} \cdots\left(\frac{d^{\alpha_{l l 1}}}{d z^{\alpha_{l l 1}}} \varphi(z)\right)^{n_{l l l}}=\varphi^{\prime}(z)^{-l}
$$

and for fixed $j$, at least one of $\beta_{l j 1}, \cdots, \beta_{l j(l-1)}$ is greater than or equal to 2 , such that

$$
\begin{aligned}
\left.\frac{d^{l}}{d w^{l}} \widetilde{f}(w)\right|_{w=\varphi(z)}= & \frac{\sqrt{\varphi^{\prime}(a)}}{\sqrt{\varphi^{\prime}(a)}} \sum_{j=1}^{l} e_{l j}\left(\frac{d^{\beta_{l j 1}}}{d z^{\beta_{l j 1}}} \varphi(z)\right)^{n_{l j 1}} \\
& \cdots\left(\frac{d^{\beta_{l j l}}}{d z^{\beta_{l j l}}} \varphi(z)\right)^{n_{l j l}} \frac{d^{j}}{d z^{j}} f_{a}(z) .
\end{aligned}
$$

For $j=1,2, \cdots, n-1$, we define the function $f_{j}$ and $\widetilde{f}_{j}$ defined in a neighborhood of $a_{j}$ in $\Omega$ and $\varphi\left(a_{j}\right)$ in $\widetilde{\Omega}$, respectively so that

$$
\begin{aligned}
f_{a}(z) & =\left(z-a_{j}\right) f_{j}(z) \text { near } z=a_{j} \\
\widetilde{f}(w) & =\left(w-\varphi\left(a_{j}\right)\right) \tilde{f}_{j}(w) \text { near } w=\varphi\left(a_{j}\right) .
\end{aligned}
$$

Hence, for a fixed $l=0,1, \cdots, m-2$, from (3.16) we have the identity

$$
\left|\left[\frac{d^{l}}{d w^{l}} \widetilde{f}_{j}(w)\right]_{w=\varphi\left(a_{j}\right)}\right|=\left|\left[\frac{d^{l}}{d z^{l}} f_{j}\right]_{z=a_{j}}\right|
$$

under the normalized condition

$$
\left|\varphi^{\prime}(a)\right|=\left|\varphi^{\prime}\left(a_{j}\right)\right|=1, \varphi^{(2)}\left(a_{j}\right)=\cdots=\varphi^{(l)}\left(a_{j}\right)=0 .
$$

Finally, in view of (3.14), (3.15), (3.18), we obtain

$$
\widetilde{c}_{j l}=c_{j l}
$$

under the same condition as (3.19) which concludes the proof of invariance of the constants $c_{j l}$. 


\section{References}

[1] Lars V. Ahlfors, Complex analysis. An introduction to the theory of analytic functions of one complex variable, McGraw-Hill Book Company, Inc., New YorkToronto-London, 1953.

[2] S. Bell, Solving the Dirichlet problem in the plane by means of the Cauchy integral, Indiana Univ. Math. J. 39(4) (1990), 1355-1371.

[3] Steve Bell, The Szegö projection and the classical objects of potential theory in the plane, Duke Math. J. 64(1) (1991), 1-26.

[4] Steven R. Bell, The Cauchy transform, potential theory, and conformal mapping, Studies in Advanced Mathematics, CRC Press, Boca Raton, FL, 1992.

[5] _ The fundamental role of the Szegö kernel in potential theory and complex analysis, J. Reine Angew. Math. 525 (2000), 1-16.

[6] Y.-B. Chung, The Ahlfors mapping function and an extremal problem in the plane, Houston J. Math. 19 (1993), 263-273.

[7] Young-Bok Chung, Higher order extremal problem and proper holomorphic mapping, Houston J. Math. 27(3) (2001), 707-718.

[8] Zeev Nehari, Conformal mapping, Dover Publications Inc., New York, 1975, Reprinting of the 1952 edition.

\section{Young-Bok Chung}

Department of Mathematics, Chonnam National University,

Gwangju 500-757, Korea.

E-mail: ybchung@chonnam.ac.kr

Byoung-Il Park

Department of Mathematics, Chonnam National University,

Gwangju 500-757, Korea.

E-mail: seraph-1@nate.com 